\title{
Modeling and forecasting hospitalized mortality rate of COVID-19 according to delay effect of daily hospitalization
}

\section{Sedighe Rastaghi}

Mashhad University of Medical Sciences

Noushin Akbari Shark

Mashhad University of Medical Sciences

Azadeh Saki ( $\sim$ azadehsaki@gmail.com )

Mashhad University of Medical Sciences https://orcid.org/0000-0002-4144-5810

\section{Research Article}

Keywords: COVID-19, mortality rate, hospitalization, Distribution Delay Model (DDM), Forecasting

Posted Date: October 19th, 2020

DOI: https://doi.org/10.21203/rs.3.rs-93952/v1

License: (c) (i) This work is licensed under a Creative Commons Attribution 4.0 International License.

Read Full License 


\section{Modeling and forecasting hospitalized mortality rate of COVID-19 according to delay effect of daily hospitalization}

\section{Abstract:}

The COVID-19 infectious epidemic has become a serious worry all over the world, including Iran. The high outbreak of disease ranked Iran as second in Asia and 11th in the world. Given the growing progress of this epidemic in infecting and killing individuals, it is essential to forecast the delay effect of the number of hospitalized upon the hospitalized mortality rate.

Methods: In this study, we used the daily Hospitalization cases of COVID-19 of IRAN for the period of 15-May 2020 to 5-Oct 2020 which were obtained from the "Iran-COVID-19-Data[1]" database. Five distribution delay models were compared for estimating and forecasting.

Results: based on measurement errors DDM selected as the best model for forecasting the number of death. According to this model, the long-run effects show that observing the effect of hospitalization counts on death counts takes an average of five days and the overall hospitalized mortality rate was $12 \%$.

\section{Conclusion:}

The overall hospitalized mortality rate of COVID-19 in Iran is less than the global rate of $15 \%$. The mean of delay effect of daily hospitalization on mortality is approximately 5 days. Our findings showed distributed delay model (DDM) has better performance in the forecasting of the future behavior of the Coronavirus mortality, and providing to government and health care decision- makers the possibility to predict the outcomes of their decision on public health.

Key words: COVID-19, mortality rate, hospitalization, Distribution Delay Model (DDM), Forecasting, 


\section{Introduction:}

The coronavirus disease epidemic began in Wuhan, China, and gradually increased worldwide, affecting large numbers of people around the world. (1, 2). The World Health Organization announced the pandemic of a worldwide epidemic, On March 11, 2020. The first coronavirus deaths in IRAN were declared on Feb 19, 2020(3). Still, the full Clinical symptoms of COVID-19 are not explicit and its exact risk of transmission is unknown. In general, everyone is at danger for this disease(4). The worst situation experienced by the USA followed by Brazil, India, Russia, and South Africa. The high outbreak of disease ranked Iran as second in Asia and 11th in the world. According to WHO, 320,117 Coronavirus Cases and 17,976 deaths in Iran, as of August 5, 2020 (5).

Due to the rapid transmission of this infection and the severity of its symptoms, the health care system is facing challenging problems and even developed countries have struggled to control it(6).

Managing worldwide epidemic such as coronavirus disease is crucial. without control with a high duplication rate (R0) measure ed at diverse immensity up to $6.5(7,8)$ and up to $14.7 \%$ of those infected requiring hospitalization $(9)$, the virus can pervade swiftly among a lot of people, Which leads to a high demand for hospital care and that the care system will not be able to handle it $(6,7)$. In such a situation, if patients do not have access to hospital services, it is likely to lead to inevitable deaths(10). notwithstanding containment policy and quarantine attempts Around the World, The prevalence of this disease is increasing and many deaths have occurred in most countries. (11).

Increasing of confirming and hospitalization cases generate outnumbered of health care facilities and other medical supplies. Hospitals, quarantine sites, ventilators, beds, Personal Protective Equipment (PPE) kits, and other medical facilities need to strengthen the health system of those counties to overcome the negative consequences of COVID 19.

In this situation, statistical models that are deferent in nature are used for forecasting but do not insist on their correctness. The results of these models can be helpful for decision-making conditions for health policymakers and authorities(12). This study aimed to model and determine the epidemic trend and predict the number of patients hospitalized due to COVID-19 in Iran using mathematical and statistical modeling.

The review of the study was focused on modeling epidemics for various destinations. The researchers have used mathematical and statistical models for the aim. Konarasinghe (2020) has modeled the epidemic of the USA, UK, and Russia by utilizing the ARIMA, Auto Regressive Distributed Delay Model (ADDM), and Double Exponential Smoothing (DES) (13). 
Konarasinghe (2020) also used The Auto-Regressive Integrated Moving Average (ARIMA) model, Autoregressive Distributed Delay Model (ADDM) Double Exponential Smoothing (DES) techniques, Liner trend model, Quadratic trend model, Growth Curve (GC) model, and Pearl - Reed Logistic model to forecast the number of infected cases within India and Brazil(14).

Soukhovolsky and et al (2020) used ADL(autoregressive distributed delay)-model on COVID-19, allows describing non-monotonic changes about infection over time (15).

This study was conducted to the forecasting future of trend epidemic the number of daily death cases COVID-19 until 27 August 2020 in Iran using the autoregressive distributed delay (ARDD) and distributed delay (DD) models. In order to show the capability of the proposed models, a comparison was made between the ARDDM and DDM model. The total number of confirmed patients and deaths in Iran was 312035 and 17405, respectively, until 3 August 2020.

Given that the number of hospital mortality rate of COVID-19 varies on a daily basis and the estimation of mortality depends on the number of daily hospitalizations, the use of conventional methods to estimate hospital mortality rate is not appropriate. In this study, an attempt has been made to estimate this rate using distribution delay models and to obtain the delay effect of hospitalization cases in the future days. Therefore, finding predictive models for forecasting mortality rate of COVID19 is a timely requirement. The study was designed to fill the knowledge gap. The objective of the study is to estimate Hospital mortality rate and forecast the number of deaths cases of COVID -19 in Iran.

\section{METHODOLOGY}

The daily Death and hospitalization cases of COVID-19 of Iran for the period of 15-May 2020 to 5-Oct 2020 were obtained from the " Iran-COVID-19-Data ${ }^{1 "}$ database.

Supported by the pattern recognition, Autoregressive Distributed Delay Model (ADDM), Distributed Delay Model, were tested to forecast the pandemic of Iran.

\footnotetext{
${ }^{1}$ https://github.com/smajidi/Iran-COVID-19-Data/blob/master/Iran_Dailly Covid19_Stat.csv
} 
The forecasting ability of the models was assessed by MAE ${ }^{2}, \mathrm{MSE}^{3}, \mathrm{MPE}^{4}, \mathrm{SMAPE}^{5}, \mathrm{MAPE}^{6}, \mathrm{MASE}^{7}, \mathrm{MRAE}^{8}, \mathrm{GMRAE}^{9}, \mathrm{MBRAE}^{10}$ and UMBRAE $^{11}$ which are measurements of errors.

Dealing with delayed effects by defining temporal construction and explicit description of delays is feasible with the time series study design. Delayed effects are explained by distributed delay models (DDMs). These models are widely used in economics(16) and lately are used in studies of environmental factors to assess health effects(17-19).

In these methods, the effect of the explanatory variable on the dependent variable occurs over future period time at deferent delays. The assumption in common DDMs is linearity among dependent and independent variables(20).

\section{Finite distributed delay models (DDM)}

In this model we supposed that the effect explanatory variables may be delayed it means that its effect occurs overtime.

In a distributed delay model the effect of an exploratory variable $x$ on y occurs over time not all at once. We can write the infinite linear DDM as follows:

$$
Y_{t}=\alpha+\sum_{s=0}^{\infty} \beta_{s} X_{t-s}+\epsilon_{t}
$$

Were $E\left(\epsilon_{t}\right)=0$ and $\operatorname{var}\left(\epsilon_{t}\right)=c, \alpha$ is the overall mean effect, $\beta$-parameters are called the delay weights and $\beta 0$ shows the effect of independent series. Estimate an infinite number of $\beta$ coefficients is impossible, and it is usually assumed that $\lim _{\mathrm{i} \rightarrow \infty} \beta_{\mathrm{i}}=0$ and $\sum_{i=0}^{\infty} \beta_{i}=\beta<\infty$.

\footnotetext{
2 mean absolute error

${ }^{3}$ mean squared error

${ }^{4}$ mean percentage error

${ }^{5}$ symmetric mean absolute percentage error

${ }^{6}$ mean absolute percentage error

${ }^{7}$ mean absolute scaled error

${ }^{8}$ mean relative absolute error

${ }^{9}$ geometric mean relative absolute error

${ }^{10}$ mean bounded relative absolute error

${ }^{11}$ unscaled MBRAE
} 
Now assume that the changes in $X_{\mathrm{t}}$ do not have much effect after a few periods of time for example, $m$, which means that all $\beta_{\mathrm{i}}$ after $\beta_{\mathrm{m}}$ disappear. In this case, the model reduces to a finite distributed delay, for which the upper limit of the summation signs in (1) is $m$.

One solution is to bounded summation to finite-length $q$, so the DDM converts to be a finite linear DDM of order q. note that model parameters estimation is based on $\mathrm{n}-\mathrm{q}$ pairs of $\left[y_{t} \cdot x_{t}\right]$. The finite distributed delay model has two disadvantages which are related to the existence of multi-collinearity and decreasing degrees of freedom with increasing delay length One important aspect to be considered is the number of parameters involved in these distributed delay models. In order to be parsimonious it is usually assumed that the coefficients of delayged variables are not all independent but functionally related.

DDMs are known as dynamic models because the effect of $x$ on $Y$ occurs over time. It means if the value of $x$ changed today the effect of this change appears today and in the future days(21-23).

\section{Autoregressive Distributed Delay Model (ARDDM)}

The autoregressive DDMs are regressions that include delays of both the dependent and independent variables. An ARDD $(p, q)$ model contains $\mathrm{p}$ delays of independent and $\mathrm{q}$ delays of dependent series. The model with $y_{t}$ as dependent, $x_{t i}$ as ith independent series, $p_{i}$ and $q$ are respectively as the delay order of ith independent series and as the autoregressive order of the model is define as follows(21):

$$
Y_{t}=\mu_{0}+\sum_{i=1}^{k} \beta_{0 i} X_{t i}+\beta_{1 i} X_{(t-1) i}+\cdots+\beta_{p i} X_{\left(t-p_{i}\right) i}+\gamma_{1} Y_{t-1}+\cdots+\gamma_{q} Y_{t-q}+e_{t}
$$

Among distributed delay models ARDD is more flexible and parsimonious(23).

\section{Implement distributed delay models with Koyck transformation (koyckDDM)}

This approach is the most general type of distributed delay model that knew as geometric DDM in which the weights of the delayged declines in a geometric pattern $\beta_{s}=\beta \phi^{s}$. $\forall \mathrm{s}$ with $0<\phi<1$. The general form of this model defines as follows:

$$
Y_{t}=\alpha+\beta\left(X_{t}+\phi X_{t-1}+\phi^{2} X_{t-2}+\phi^{3} X_{t-3}+\cdots\right)+\epsilon_{t}
$$


This model has some restrictions to implement due to the infinite number of parameters and nonlinearity. One solution to deal with these restrictions is koyck transformation. After this transformation we have finite DDM. In order to fit the model instrumental variables used. The geometric DDM with koyck transformation is as follows:

$$
Y_{t}-\phi Y_{t-1}=\alpha(1-\phi)+\beta X_{t}+\left(\epsilon_{t}-\phi \epsilon_{t-1}\right) \quad \rightarrow \quad Y_{t}=\gamma_{1}+\gamma_{2} Y_{t-1}+\gamma_{3} X_{t}+v_{t}
$$

where $\delta_{1}=\alpha(1-\phi), \delta_{2}=\phi, \delta_{3}=\beta$ and $v_{t}=\left(\varepsilon_{t}-\phi \varepsilon_{t-1}\right)$ shows the random error after the transformation. In this model, the first delay of the dependent series $y_{t-1}$ is correlated with the error term and the error term $v_{t}$ depends on both $\varepsilon_{t}$ and $\varepsilon_{t-1} \cdot(21,23)$

\section{Implement finite polynomial distributed lag model (polyDlm)}

Finite distributed lag models, in general, suffer from the multi-collinearity due to the inclusion of the lags of the same variable in the model. To reduce the impact of this multi-collinearity, a polynomial shape is imposed on the lag distribution (Judge and Griffiths, 2000). The resulting model is called the Polynomial Distributed Lag model or Almond Distributed Lag Model.

Imposing a polynomial pattern on the lag distribution is equivalent to representing $\beta$ parameters with another $\$ k \$ t h$ order polynomial model of time. So, the effect of change in $X_{t-s}$ on the expected value of $Y_{t}$ is represented as follows:

$$
\frac{\partial E\left(Y_{t}\right)}{\partial X_{t-s}}=\beta_{s}=\gamma_{0}+\gamma_{1} s+\gamma_{2} s^{2}+\cdots+\gamma_{k} s^{k}
$$

where $s=0, \ldots, q$ (Judge and Griffiths, 2000). Then the model becomes (23):

$$
Y_{t}=\alpha+\gamma_{0} Z_{t 0}+\gamma_{1} Z_{t 1}+\gamma_{2} Z_{t 2}+\cdots+\gamma_{k} Z_{t k}+\epsilon_{t}
$$




\section{Results:}

We applied distributed delay models to forecast the effect of hospitalization counts on the death count. All the models mentioned above were fitted to covid-19 data and better models for forecasting were selected Based on the error measurements.

Table1 shows the nine measurements of errors for fitted models. According to the value of these indexes ARDDM and DDM models had minimum errors and selected for forecasting the number of death.

Table 2 shows the results of fitting RDDM model. Base on this model the median delay 0.04 , indicates that at the end of the fifth day at least half of the effect of hospitalizations number can be seen on the number of deaths.

The long-run effect of the number of hospitalizations on the number of deaths is 0.0883 and it takes an average of seventh days to observe the hospitalization counts on the death counts. It means if the number of hospitalizations at the beginning of the study was 1000 people, we will see 88 deaths by the end of the seventh day due to the long-run effect. Duo to the median delay, half of this number of the death will be observed in the first five days ( 43 people and approximately 8.8 people per day) and the other half of this number of deaths will occur on the sixth and seventh day (44 deaths on the sixth and seventh day, ie 22 deaths almost every day).

Table 3 shows the results of fitting DDM model. Base on this model the median delay 0.061 , shows that at the end of the fifth day at least half of the effect of hospitalizations counts can be seen on the number of deaths.

The long-run effect of the number of hospitalizations on the number of deaths is 0.123 and it takes an average of five days to observe the hospitalization counts on the death counts. It means if the number of hospitalizations at the beginning of the study was 1000 people, we will see 123 deaths by the end of the seventh day due to the long-run effect. Duo to the median delay, half of this number of the death will be observed in the first five days (60 people and approximately 12 people per day) and the other half of this number of deaths will occur on the sixth and seventh day (60 deaths on the sixth day and Seventh, which means 30 deaths almost every day).

Table 4 shows the forecasting number of death for different sets of hospitalization numbers on based ARDDM and DDM models. We define three trend scenarios for hospitalization counts: increasing, decreasing, and constant. In both ARDDM and DDM models predicted the death counts were almost identical and show an upward trend for both increasing and constant situations. 
Table1: forecasting evaluation criteria ,AIC and Adjusted R-squared for distributed delay models.

\begin{tabular}{|c|c|c|c|c|c|c|c|c|c|c|c|c|c|}
\hline & $\mathrm{n}$ & MAE & MPE & MAPE & SMAPE & MASE & MSE & MRAE & GMRAE & MBRAE & UMBRAE & AIC & $\begin{array}{c}\text { Adjusted R- } \\
\text { squared }\end{array}$ \\
\hline DDM & 136 & 13.27 & -0.01 & 0.10 & 0.10 & 0.88 & 259.98 & 1.92 & 0.95 & 0.55 & 1.24 & 1162.19 & 0.892 \\
\hline ARDD model & 136 & 12.33 & -0.01 & 0.09 & 0.09 & 0.82 & 234.19 & 1.51 & 0.85 & 0.57 & 1.34 & 1149.99 & 0.902 \\
\hline koyck model & 142 & 13.64 & -0.02 & 0.11 & 0.10 & 0.92 & 331.84 & 1.54 & 0.87 & 1.05 & -22.32 & 1235.24 & 0.878 \\
\hline polyDDM model & 136 & 13.32 & -0.01 & 0.10 & 0.10 & 0.88 & 261.24 & 1.91 & 0.96 & 0.12 & 0.14 & 1152.85 & 0.895 \\
\hline
\end{tabular}

Table2: results of fitting RDDM model

\begin{tabular}{|c|c|c|c|c|c|c|c|c|c|c|c|}
\hline & & & Estimate & Std. Error & $\mathrm{t}$ value & $\operatorname{Pr}(>|t|)$ & & & $\begin{array}{c}\text { Median del } \\
\frac{1}{2} \sum_{\mathrm{j}=0}^{7} \alpha_{\mathrm{j}}\end{array}$ & $\begin{array}{l}\text { Long-run } \\
\sum_{j=0}^{7} \alpha_{j} \\
=\sum_{j=0}^{7} \beta_{j} \\
+\alpha_{j-1} \gamma_{1}\end{array}$ & $\begin{array}{c}\text { Instant } \\
\text { effect } \\
\beta_{0}=\alpha_{0}\end{array}$ \\
\hline \multirow{6}{*}{$\begin{array}{c}\text { Autoregressive } \\
\text { distributed delay } \\
\text { models }\end{array}$} & & (Intercept) & 4.234 & 4.400 & 0.962 & 0.338 & & & \multirow{6}{*}{0.044138} & \multirow{6}{*}{0.0883} & \multirow{6}{*}{0.009108} \\
\hline & $\beta_{0}$ & hospitalize.t & 0.009 & 0.008 & 1.178 & 0.241 & $\alpha_{0}$ & 0.0091 & & & \\
\hline & $\beta_{1}$ & hospitalize .1 & 0.006 & 0.009 & 0.685 & 0.495 & $\alpha_{1}$ & 0.0083 & & & \\
\hline & $\beta_{2}$ & hospitalize .2 & -0.002 & 0.009 & -0.255 & 0.799 & $\alpha_{2}$ & -0.0022 & & & \\
\hline & $\beta_{3}$ & hospitalize .3 & 0.009 & 0.009 & 1.016 & 0.312 & $\alpha_{3}$ & 0.0087 & & & \\
\hline & $\bar{\beta}$ & hospitalize .4 & 0.008 & 0.009 & 0.871 & 0.385 & $\alpha_{4}$ & 0.0075 & & & \\
\hline
\end{tabular}




\begin{tabular}{|c|c|c|c|c|c|c|c|c|c|}
\hline$\beta_{5}$ & hospitalize .5 & 0.020 & 0.009 & 2.231 & $0.027483^{*}$ & $\alpha_{5}$ & 0.0195 & \\
& $\beta_{6}$ & hospitalize .6 & 0.018 & 0.009 & 1.996 & $0.048144^{*}$ & $\alpha_{6}$ & 0.0185 \\
\cline { 2 - 8 } & $\beta_{7}$ & hospitalize .7 & 0.019 & 0.009 & 2.180 & $0.031110^{*}$ & $\alpha_{7}$ & 0.0189 & \\
\cline { 2 - 7 } & $\gamma_{1}$ & death.1 & 0.301 & 0.081 & 3.725 & $0.000294^{* * *}$ & & \\
\hline
\end{tabular}

Table3: results of fitting DDM model

\begin{tabular}{|c|c|c|c|c|c|c|c|c|}
\hline & & estimate & eroor & t-value & $p$-value & Median delay & Mean delay & Long-run \\
\hline \multirow{9}{*}{ DDM } & intercept & 6.051963 & 4.589445 & 1.319 & 0.189652 & \multirow{9}{*}{0.061} & \multirow{9}{*}{4.653} & \multirow{9}{*}{0.123} \\
\hline & hospitalize.t & 0.010990 & 0.008097 & 1.357 & 0.177068 & & & \\
\hline & hospitalize .1 & 0.008728 & 0.009094 & 0.960 & 0.338964 & & & \\
\hline & hospitalize .2 & 0.002667 & 0.008948 & 0.298 & 0.766193 & & & \\
\hline & hospitalize .3 & 0.010689 & 0.008973 & 1.191 & 0.235795 & & & \\
\hline & hospitalize .4 & 0.011352 & 0.008986 & 1.263 & 0.208796 & & & \\
\hline & hospitalize .5 & 0.021365 & 0.009182 & 2.327 & $0.021559 *$ & & & \\
\hline & hospitalize .6 & 0.025504 & 0.009500 & 2.685 & $0.008228 * *$ & & & \\
\hline & hospitalize .7 & 0.031257 & 0.008398 & 3.722 & $0.000296 * * *$ & & & \\
\hline
\end{tabular}


Table4: forecasting number of the death based on the different set of hospitalization numbers

\begin{tabular}{|c|c|c|c|c|c|c|c|c|}
\hline hospitalize & forecast.DDM & forecast.RDDM & hospitalize & forecast.DDM & forecast.RDDM & hospitalize & forecast.DDM & forecast.RDDM \\
\hline 1950 & 218.7 & 225.6 & 1950 & 218.7 & 225.6 & 1950 & 218.7 & 225.6 \\
\hline 1950 & 210.4 & 214.0 & 2000 & 210.9 & 214.4 & 1900 & 209.8 & 213.5 \\
\hline 1950 & 214.3 & 212.2 & 2050 & 215.9 & 213.6 & 1850 & 212.8 & 210.9 \\
\hline 1950 & 205.2 & 206.9 & 2100 & 207.9 & 209.2 & 1800 & 202.6 & 204.7 \\
\hline 1950 & 216.0 & 215.2 & 2150 & 220.3 & 218.8 & 1750 & 211.7 & 211.6 \\
\hline 1950 & 230.5 & 227.9 & 2200 & 237.0 & 233.4 & 1700 & 224.0 & 222.4 \\
\hline 1950 & 245.1 & 240.5 & 2250 & 254.9 & 249.0 & 1650 & 235.3 & 232.1 \\
\hline 1950 & 245.0 & 244.3 & 2300 & 259.4 & 257.0 & 1600 & 230.6 & 231.5 \\
\hline 1950 & 245.0 & 245.4 & 2350 & 265.5 & 263.7 & 1550 & 224.5 & 227.1 \\
\hline 1950 & 245.0 & 245.7 & 2400 & 271.7 & 270.0 & 1500 & 218.4 & 221.5 \\
\hline 1950 & 245.0 & 245.8 & 2450 & 277.8 & 276.2 & 1450 & 212.3 & 215.5 \\
\hline 1950 & 245.0 & 245.9 & 2500 & 283.9 & 282.4 & 1400 & 206.1 & 209.4 \\
\hline 1950 & 245.0 & 245.9 & 2550 & 290.0 & 288.5 & 1350 & 200.0 & 203.2 \\
\hline 1950 & 245.0 & 245.9 & 2600 & 296.2 & 294.7 & 1300 & 193.9 & 197.1 \\
\hline
\end{tabular}

\section{Discussion:}

According to the value of errors for fitted models ARDDM, and DDM models had minimum errors respectively. with fitted models we found that at the end of the fifth day of hospitalization, at least half of the effect of the hospitalization counts could be observed on the death counts. 
Mathematical and statistical models provided by scientists can help significantly in identifying the course of the disease outbreak as well as identifying effective health interventions in reducing the progression of epidemic progression. There are three common approaches to such modeling. The first method is a nonlinear regression model such as the agent-based model (24).

These methods make it possible to accurately estimate based on given data as well as short-term predictions for incidence, but describing the mechanism of the pandemic is difficult to describe the based on them. SIR is the most common model used for coronaviruses spread such as SEIR-models(25-27). In these models, there is a challenge in estimating S-group.

Based on the SIR-model, by adopting that the entire population of the country should be in this group, then the outbreak will stop just if the Majority of people have been infected $(28,29)$. Furthermore, the SIR-models do not beg the question of the critical point of People infected, after reaching the early exponential phase. When choosing a model with large enough initial values, it is difficult to answer this question.

Eventually, a recurrent bifurcation model was proposed, which showed that disease transmission is a pandemic even in a small number of cases. (30). This procedure is similar to the method we have proposed.

This paper investigates impact Number of hospitalizations and time delay Number of hospitalizations of COVID-19 pandemic (new cases and new deaths) on the IRAN Number of deaths. For this purpose, Autoregressive-Distribute Delay (ARDD) and Distribute Delay (DDM) model have been applied to the data from 15-May 2020 to 3-Aug 2020. In order to obtain short and long-term effects on the number of deaths

From the outcome of the study based on DDM, hospitalizations have a long-run influence on new deaths. However, the relationship is not strong in the short-term. For controlling this dangerous state, the results of this study can be of great help to policymakers.

Based on forecasting results DDM was better than ARDD and also the overall estimate of death based on DDM was closer to a global standard that is 15 percent. For this purpose, Autoregressive-Distribute Delay (ARDD) and Distribute Delay (DDM) model have been applied to the data from 15-May 2020 to 3-Aug 2020 in order to capture both long-run and short-run impact on a number of deaths.

\section{Conclusion}

From a data point of view, this model is very simple and practical for calculations, so only data from the current rate is sufficient to confirm the proposed model. However, the coefficient of determination of the R2 model is very high. Given this, we predict hospital mortality for up to 14 days.

The ADL model allows describing non-monotonic changes about infection over time, and assists governments and health policy makers in anticipating and making appropriate health decisions. 


\section{Author contributions}

AS Have made substantial contributions to conception and design; SR and NA contributed in statistical analysis and wrote the manuscript; AS critically reviewed the manuscript.

\section{Competing Interests}

There is no competing interest.

\section{Ethical Approval}

Ethical approval was not required as this study made use of publicly available data.

\section{Funding}

None. 


\section{Reference:}

1. Tuite AR, Bogoch II, Sherbo R, Watts A, Fisman D, Khan K. Estimation of coronavirus disease 2019 (COVID-19) burden and potential for international dissemination of infection from Iran. Annals of Internal Medicine. 2020;172(10):699-701.

2. da Silva CAG. A CLASSIFICATION STUDY OF BRAZIL 'S FEDERATIVE UNITS FOR COVID-BASED IN HIDDEN MARKOV MODEL: THE NEW EPICENTER OF SARS-COV-VIRUS IN THE WORLD.

3. Takian A, Raoofi A, Kazempour-Ardebili S. COVID-19 battle during the toughest sanctions against Iran. Lancet (London, England). 2020;395(10229):1035.

4. $\quad$ Adhikari SP, Meng S, Wu Y-J, Mao Y-P, Ye R-X, Wang Q-Z, et al. Epidemiology, causes, clinical manifestation and diagnosis, prevention and control of coronavirus disease (COVID-19) during the early outbreak period: a scoping review. Infectious diseases of poverty. 2020;9(1):1-12.

5. https://www.worldometers.info/coronavirus/?utm campaign=homeAdvegas1?

6. Grasselli G, Pesenti A, Cecconi M. Critical care utilization for the COVID-19 outbreak in Lombardy, Italy: early experience and forecast during an emergency response. Jama. 2020;323(16):1545-6.

7. Ferguson N, Laydon D, Nedjati-Gilani G, Imai N, Ainslie K, Baguelin M, et al. Report 9: Impact of non-pharmaceutical interventions (NPIs) to reduce COVID19 mortality and healthcare demand. Imperial College London. 2020;10:77482.

8. Liu Y, Gayle AA, Wilder-Smith A, Rocklöv J. The reproductive number of COVID-19 is higher compared to SARS coronavirus. Journal of travel medicine. 2020.

9. Docherty AB, Harrison E, Green C. Features of 16,749 hospitalised UK patients with COVID-19 using the ISARIC WHO Clinical Characterisation Protocol. medRxiv. The Preprint Server for Health Science. 2020;28.

10. White DB, Lo B. A framework for rationing ventilators and critical care beds during the COVID-19 pandemic. Jama. 2020;323(18):1773-4.

11. Anderson RM, Heesterbeek $\mathrm{H}$, Klinkenberg $\mathrm{D}$, Hollingsworth TD. How will country-based mitigation measures influence the course of the COVID-19 epidemic? The Lancet. 2020;395(10228):931-4.

12. Hellewell J, Abbott S, Gimma A, Bosse NI, Jarvis Cl, Russell TW, et al. Feasibility of controlling COVID-19 outbreaks by isolation of cases and contacts. The Lancet Global Health. 2020.

13. Konarasinghe K. Modeling COVID-19 Epidemic of USA, UK and Russia. Journal of New Frontiers in Healthcare and Biological Sciences. 2020;1(1):1-14.

14. Konarasinghe KMUB. Modeling COVID -19 Epidemic of India and Brazil. Journal of New Frontiers in Healthcare and

Biological Sciences. 2020;1.

15. Soukhovolsky V, Kovalev A, Pitt A, Kessel B. A new modelling of the COVID 19 pandemic. Chaos, Solitons \& Fractals. 2020:110039.

16. Almon S. The distributed lag between capital appropriations and expenditures. Econometrica: Journal of the Econometric Society. 1965:178-96.

17. Schwartz J. The distributed lag between air pollution and daily deaths. Epidemiology. 2000;11(3):320-6. 
18. Zanobetti A, Wand MP, Schwartz J, Ryan LM. Generalized additive distributed lag models: quantifying mortality displacement. Biostatistics. 2000;1(3):279-92.

19. Muggeo VM, Hajat S. Modelling the non-linear multiple-lag effects of ambient temperature on mortality in Santiago and Palermo: a constrained segmented distributed lag approach. Occupational and Environmental Medicine. 2009;66(9):584-91.

20. Gasparrini A. Distributed lag linear and non-linear models in R: the package dlnm. Journal of statistical software. 2011;43(8):1.

21. Demirhan H. dLagM: An R package for distributed lag models and ARDL bounds testing. Plos one. 2020;15(2):e0228812.

22. R. Ravines R, M. Schmidt A, S. Migon HJASMiB, Industry. Revisiting distributed lag models through a Bayesian perspective. 2006;22(2):193-210.

23. Demirhan H. dLagM: Time Series Regression Models with Distributed Lag Models. R package version. 2019;1:12.

24. Tuomisto JT, Yrjölä J, Kolehmainen M, Bonsdorff J, Pekkanen J, Tikkanen TJm. An agent-based epidemic model REINA for COVID-19 to identify destructive policies. 2020.

25. Clifford SJ, Klepac P, Van Zandvoort K, Quilty BJ, Eggo RM, Flasche S, et al. Interventions targeting air travellers early in the pandemic may delay local outbreaks of SARS-CoV-2. 2020.

26. Shen M, Peng Z, Guo Y, Xiao Y, Zhang Lm. Lockdown may partially halt the spread of 2019 novel coronavirus in Hubei province, China. 2020.

27. Tang B, Bragazzi NL, Li Q, Tang S, Xiao Y, Wu JJIdm. An updated estimation of the risk of transmission of the novel coronavirus (2019nCov). 2020;5:248-55.

28. Allali M, Portecop P, Carlès M, Gibert DJm. Prediction of the time evolution of the COVID-19 disease in Guadeloupe with a stochastic evolutionary model. 2020.

29. Ediriweera DS, de Silva NR, Malavige NG, de Silva HJJm. AN EPIDEMIOLOGICAL MODEL TO AID DECISION-MAKING FOR COVID-19 CONTROL IN SRI LANKA. 2020.

30. Shen JJm. A Recursive Bifurcation Model for Predicting the Peak of COVID-19 Virus Spread in United States and Germany. 2020. 